\title{
ON THE FATE OF THE JUGULAR LYMPH SACS AND THE DEVELOPMENT OF THE LYMPH CHAN- NELS IN THE NECK OF THE PIG
}

\author{
ADMONT H. CLARK \\ From the Anatomical Laboratory, Johns Hopkins University \\ FOUR FIGURES
}

In a study of the morphological changes which the jugular lymph sacs and the lymph channels in the neck of the embryo pig undergo during development, a number of questions must be considered. What are the primary lymph channels? Are they characteristic and constant in form? How are they modified during development? What is the correlation between the earliest-lines of drainage and the drainage found in the adult? What are some of the factors controlling these transformations? These and other questions arise. The purpose of the following paper is to make an analysis, and to offer a few suggestions on the points mentioned above.

The undertaking of this work was suggested by Dr. Sabin, and it was through her kindness that this study was possible. There have been accumulating in the laboratory from previous studies a number of injections of lymphatics in embryo pigs of all stages made by Dr. Sabin. These with numerous new injections have been cleared by the Spalteholz method, ${ }^{1}$ and the present paper is based on a comparative analysis of these specimens. It has been the aim to give as accurately as possible the location of the lymphatics and the morphological changes in successive stages of development. There has been no attempt to describe the minute structure of the lymphatics but simply to trace the gross changes in the lymph channels.

\footnotetext{
${ }^{1}$ Spaltehola, W., Ueber das Durchsichtigmachen von menschlichen und tieri-
} schen Präparaten. Leipzig. Verlaz von S. Hirzel. 1911. 
The work of Sabin has shown that lymphatics first appear in the embryo pig 10 to $11 \mathrm{~mm}$. long as an outbudding from the anterior cardinal veins opposite the third, fourth and fifth segmental branches. From these primitive buds a plexus of lymphaties is formed along the dorsolateral border of the anterior cardinal vein and this plexus is transformed into a non-muscular endothelial lined sac. From this primitive sac by continued centrifugal growth a large number of sprouts grow dorsalward into the pos terior triangle of the neck and form a complete arch of lymphatic capillaries connecting at either end with the primitive sac. This entire arch of capillaries becomes transformed into a part of the jugular lymph sac which explains the form of the final sac as shown in figure 1. From the jugular sac, still by centrifugal growth, the peripheral lymph vessels radiate forward over the head and backward over the anterior part of the body forming plexuses which are characteristic and definitely located. ${ }^{2}$

For convenience I shall use the following terms in referring to the lymph sac, the form of which is shown in figure 1. (1) The anterior curvature of the lymph sac is the portion lying behind the pharnyx against the internal jugular vein. (2) The sac stalk is the portion of the sac also on the internal jugular vein extending between the point where the valve develops at the junction of the internal and external jugular veins and the anterior curvature. This is the first part of the sac to develop. The apex is the portion of the sac lying in the posterior triangle of the neck. The reasons for this division of the sac are not obvious in figure 1, but I shall show that they correspond to the function of the three different parts of the sac. The apex of the sac connects with the sac stalk both through the anterior curvature and more directly by a large channel which joins the stalk not far from the valve into the vein.

The form of the jugular sae is well shown in figure 1 , which is a diagram made from an embryo pig $2.8 \mathrm{~cm}$. long and in figure 2 which is a drawing of an injection of the lymphatics in a pig 3.5 $\mathrm{cm}$. long. From the sac four groups of lymphatic vessels develop.

${ }^{2}$ A part of this work is in the Amer. Jour. Anat., vol. 1, 1901-1902, and a part of it will be published in the Ergebnisse für Anat. und Entwicklungsgeschichte. 
The first group consists of a few large vessels which have grown from the apex, the most superficial part of the sac, over the scapular region. The second place of growth is the dorsal border of

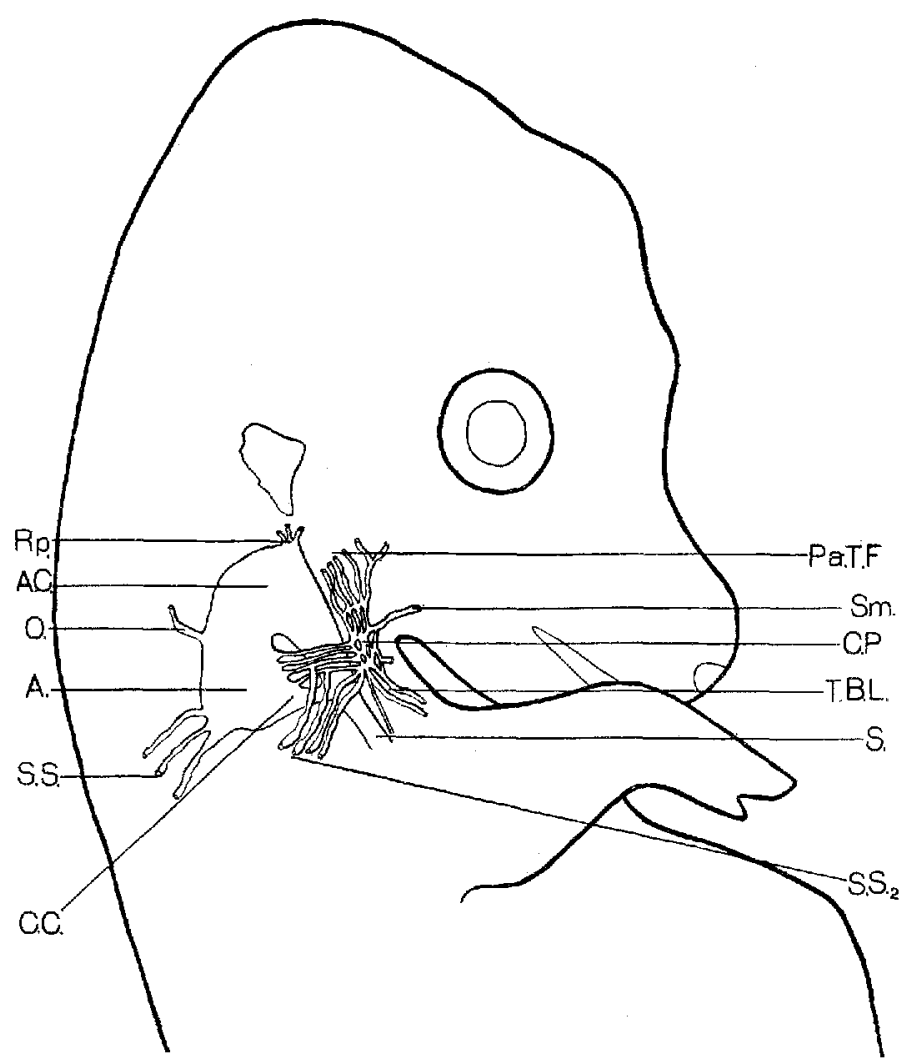

Fig. 1 Diagram of the jugular lymph sac in an cmbryo pig $2.8 \mathrm{~cm}$. long to show the points of origin of the peripheral vessels. $\times 10$. A, apcx; A.C, anterior curvature; C.C, cross connection between the apex of the sac and the sac stalk; C.P. superficial cervical plexus; $O$, occipital lymph duct; $P a . T . F$, point of origin of the posterior-auricular, temporal and facial lymphaties; $R p$, retropharyngeal lymphatics; $S$, stalk of the sec; $S m$, submaxillary lymphaties; $S . S$, primary suprascapular lymphatics; $S . S_{2}$, supraseapular tymphatics from the cervical plexus; $T . B . L$, thoracic and branchial lymphatics.

the apex just anterior to the suprascapular vessels. A large duct extends forward over the occipital region of the head. This particular vessel is very large in the human embryo as can be seen 
in figures 505 and 506 of the Handbuch der Entwickelungsgeschichte des Menschen. Keibel and Mall., vol. 2, 1911, ps. 708-709, after Sabin. The third group of vessels is from the anterior curvature of the sac where it arches dorsalward and lateralward behind the pharynx. The importance of these vessels is shown both by their size and their early appearance. In an embryo 2.8 $\mathrm{cm}$. long the anterior curvature has a distinet bulge protruding toward the buccal cavity, and in one specimen a few ducts can be seen radiating toward the pharynx. From this retropharyngeal process of the sac are to be developed all of the lymphatics of the pharynx, Eustachian tube, the nasal cavity and a part of those of the tongue. The fourth group of vessels is by far the largest. In figure 2 will be seen a group of vessels from the ventral border of the apex of the sac which grow ventralward external to the sterno-cleido-mastoid muscle and form an extensive Iymphatic plexus along the course of the external jugular vein. This plexus I shall call the superficial cervical plexus since it gives rise to the superficial cervical lymph glands.

The injection shown in figure 2 is not a complete injection for these vessels. The point of injection was in the suprascapular vessels which is an indirect point for the superficial cervical plexus. The vessels from the ventral border of the apex of the sac are present in an embryo $18 \mathrm{~mm}$. long and hence they begin at about the same time as the suprascapular lymphatics. The superficial cervical plexus as shown in figure 2 has already sent a group of vessels cranialward, part of which are shown as posterior auricular lymphatics. The vessels which grow forward along the external jugular vein divide into two groups, the temporal and the facial. From the ventral border of the superficial cervical plexus develop

Fig. 2 Injection of the jugular lymph sac in an embryo pig measuring $3.5 \mathrm{~cm}$. long. Magnified about 10 times. This is the same specimen which was shown as figure 3 in The American Journal of Anatomy, p. 186, vol. 3, 1904. The specimen has since been cleared by the Spalteholy method so that it shows the relation of the superfieial lymphaties to the jugular lymph sae. It is a complete injection of the suprascapular and occipital plexuses and an incomplete injection of the begining cervical plexus. The sac stalk shows faintly where it extends internal to the arm. F.v, facial vein; a little blood in this vein enables one to locatc the position of the superficial cervical lymphatic plexus. 


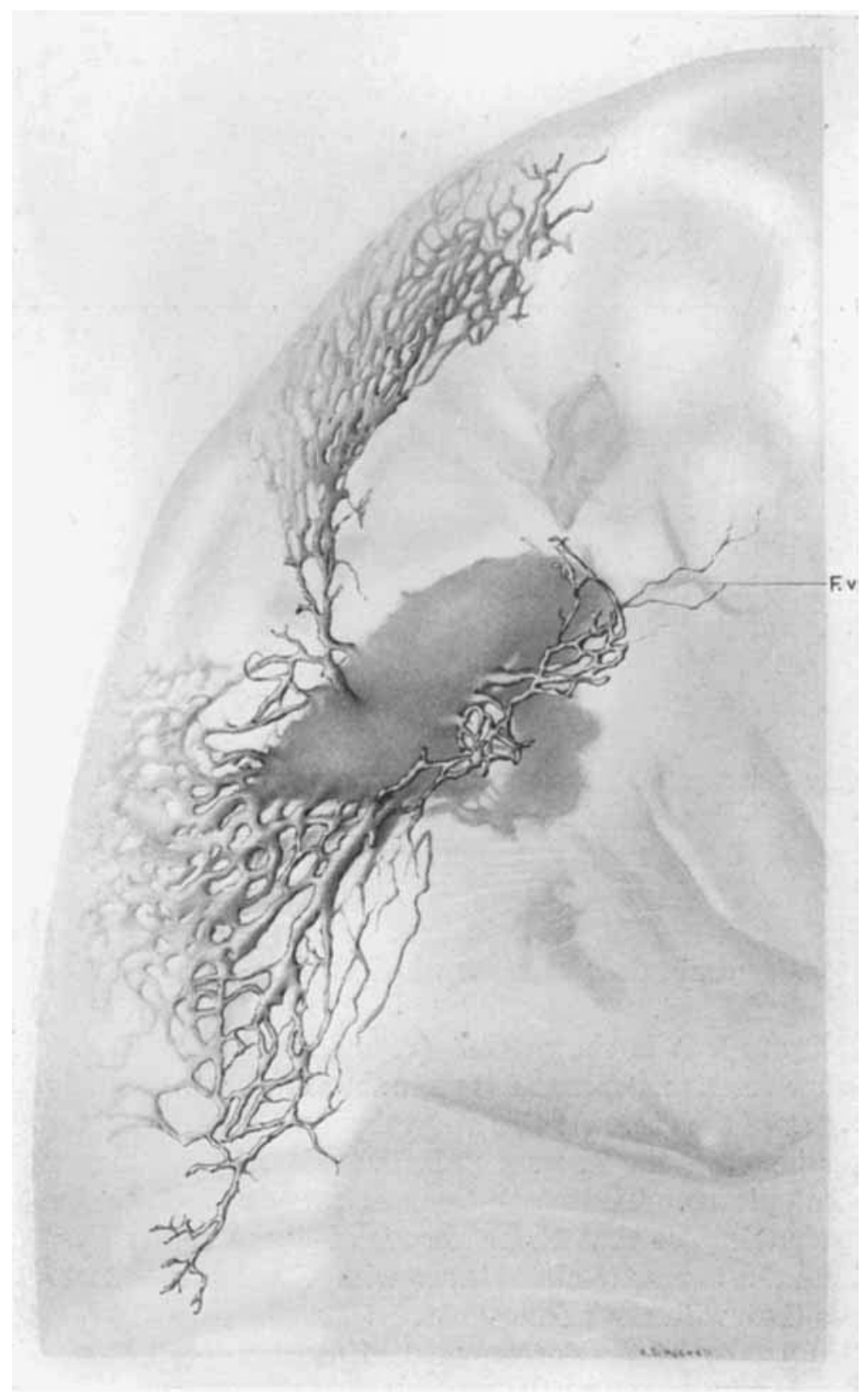


as shown in figure 1 the lymphaties for the skin of the nexk and the submaxillary vessels, while the caudal end of the plexus gives rise to the superficial lymphaties of the arm and of the thoracic wall. Thus the jugular lymph sac gives rise to the suprascapular, occipital and pharyngeal lymphaties directly and is the place of origin of the superficial cervical plexus which in turn supplies all the rest of the lymphatics for the head, face, neck, thorax and arm. The deep lymphatics of the arm have not yet been worked out in the pig. In the cat and in human embryos they arise from an extension of the jugular lymph sac which lies along the primitive ulnar vein.

These fundamental groups of lymphaties the suprascapular, occipital and superficial cervical, which can be scen in the embryo $3.5 \mathrm{~cm}$. long and indeed can be injected a short distance from the lymph sac much earlier namely in specimens measuring 18 $\mathrm{mm}$. are constant. Ducts originate from definite places and establish definitely-localized plexuses. Thus in the early stages there are distinct plexuses in the skin which are connected with each other only through their central connection with the sac. Such a primary plexus for example is the occipital plexus of figure 2. By subsequent development however, these separate areas become interconnected, so that an injection instead of being limited to one of the primary plexuses spreads out quite widely, reaching the sae not by a single set of ducts but by a number according to the extent of the injection. Thus, plainly, the earliest lymphatics drain definite areas which are distinctly located and definitely defined.

In figure 2 it is shown that the suprascapular vessels drain by a few vessels sometimes not more than one or two directly into the apex of the sac. At this stage there are a few small anastomoses between the suprascapular vessels and the superficial ccrvical plexus. These anastomoses are destined to become very abundant so that there are eventually more vessels which connect the suprascapular plexus with the superficial cervical plexus than with the primary sac.

This process of the development of anastomoses between the different primary plexuses goes on until the entire superficial 
lymphatic plexus is a complete layer of lymphatics covering the body. This stage is shown in Sabin's figure 5 in The American Journal of Anatomy, p. 188, volume 3, 1904 In this figure it is not possible to analyzo the primary plexuses; the suprascapular, oceipital, posterior auricular, temporal, facial, cervical, thoracic and brachial vessels make one continuous plexus. When an injected specimen of this stage is cleared however by the Spalteholz method the place of origin for each plexus can be made out. In figure 3 it is clear that the primary lymph sac has the three divisions already given, namely, the epex in the posterior triangle the anterior curvature and the sac stalk lying deeper and hence showing very faintly on the internal jugular vein. The apex of the sac and the anterior curvature may now be called lymph glands, the sac stalk however remains as the deep jugular lymph trunks. In comparing figures 2 and 3 it is clear that in the earlier stage the outline of the sac is a comparatively smooth curve from the stalk around to the apex. In an embryo pig $20 \mathrm{~mm}$. long the cntire dorsal border of the sac has a series of sprouts but the permanent ducts are however limited to certain areas along the sac and these parts enlarge while the intermediate parts remain small. This detcrmines the position of the lymph nodes.

Three factors secm to guide this primary node formation in the sac. First the apex of the sac receiving as it docs the suprascapular and occipital vessels directly and all of the vcssels of the face, neck, arm and thorax indirectly through the superficial cervical plexus is the largest center of drainage in the neck. Lymph glands develop at the centers of drainage and the apex of the sac therefore becomes an early and a large node. Second the portion of the sac between the apex and the anterior curvature can be assumed to be comparatively non-functional as a path for lymph conduction, for the apical drainage would most casily pass to the veins by way of the cross connection to the stalk. Hence the portion of the sac intervening between the apex and the anterior curvature remains small. Third, the development of the sternocleido-mastoid muscle which crosses the sac between the apex and the anterior curvature probably causes a pressure to be exerted at this point. Drainage, function and structural relations can 
be said to be factors in controlling node formation and development both in the lymph sac and along the peripheral lymphatics. The relations of the peripheral lymphaties to the sac and the development of nodes thus described are constant.

The superficial cervical plexus needs a very careful description. It is clear that it is an important structure since it drains so large

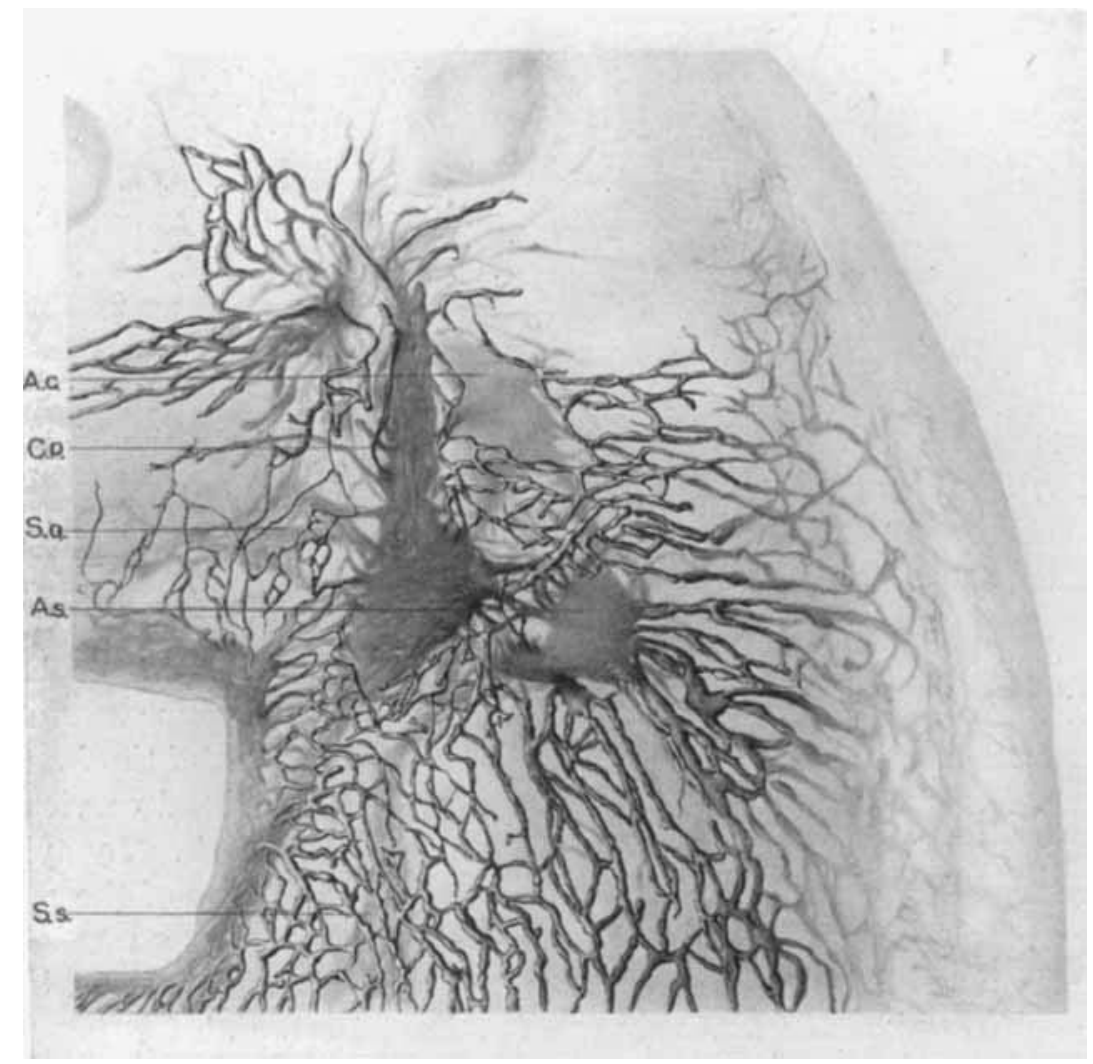

Hig. 3 Injocted jugular Jymph sac, superficial cervical lymph plexus and the peripheral lymphatics in the neck of an embryo pig moasuring $5.5 \mathrm{~cm}$. long. Magnified about 7.5 times. This figure is to be compared with figure 5 in the American Journal of Anatomy, p. 188, vol. 3, 1904, which is a complete injection of the superficial lymphatics of the same stage. A.s, the apex of the jugular sac making the lymph gland of the posterior triangle; $A . c$, anterior curvature of the lymph sac making the deep jugular lymph gland; C.p, superficial corvical lymph plexus; $S . g$, submaxillary lymph gland; $S . s$, stalk of the jugular lymph sac. 
an area. As seen in figures 3 and 4 it is an extensive and dense plexus of lymphatics lying along the external jugular vein lateral to the sterno-cleido-mastoid muscle. It has in reality two points of origin. First, the ventral border of the apex of the sac shown in figure 2 for an earlier stage but still better in figure 3 . The second place of origin is a plexus of lymphatics from the stalk of

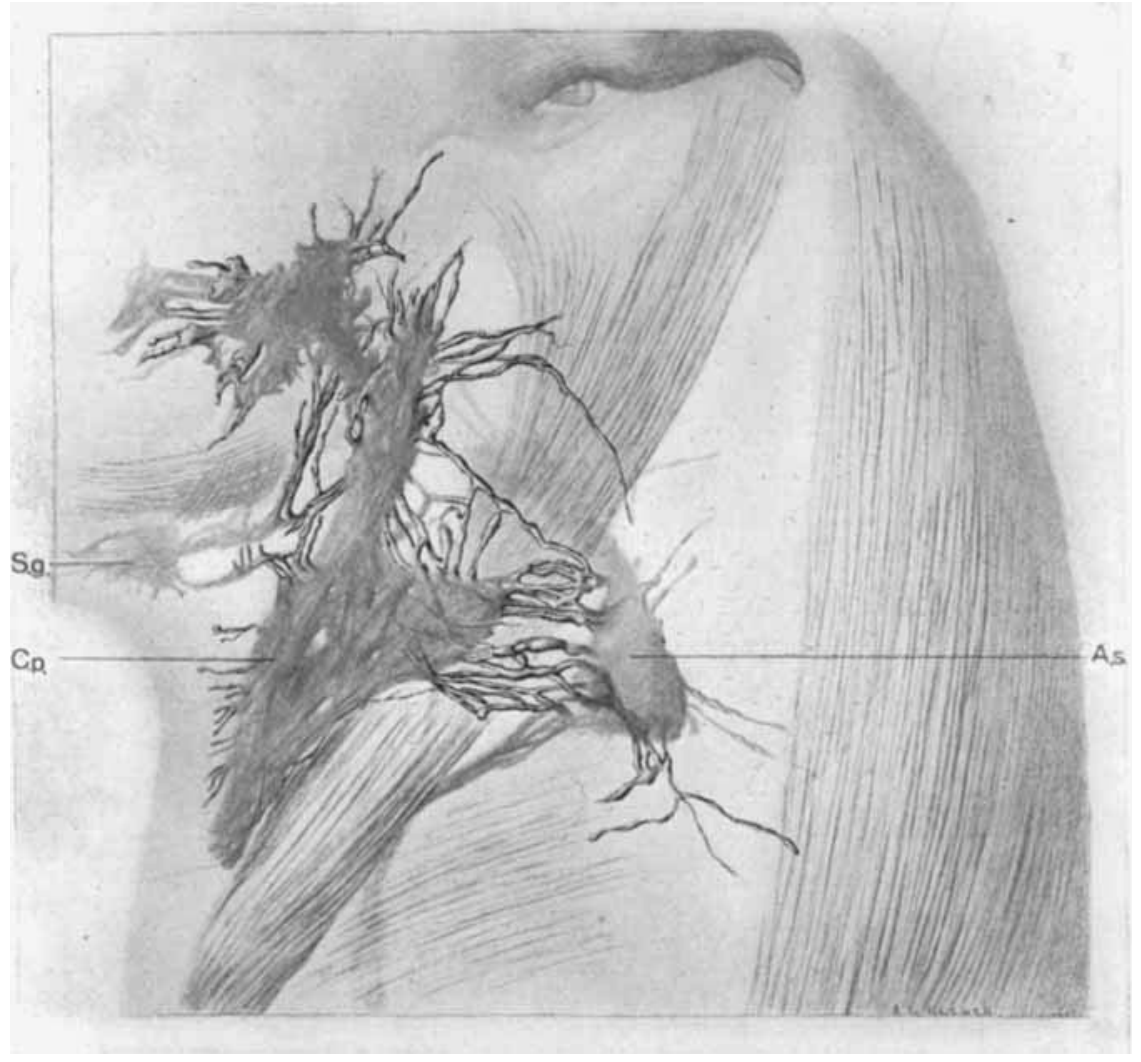

Fig. 4 Injected jugular lymph sac and cervical lymph plexus in a pig measuring $7.5 \mathrm{~cm}$. long, to show the relation of the developing glands in the neck to the sac. Magnified 6.5 times. A.s, apex of the sac or gland of the posterior triangle of the neck. The anterior curvature of the sac, which is a deep jugular pharyngeal lymph gland, shows behind the sterno-cleido-mastoid muscle. C.p, superficial cervical plexus which is destined to be a group of lymph glands. At the ccrebral end of the plexus is a developing facial gland. S.g, submaxillary lymph gland. 
the sac which follow the external jugular vein. This group of vessels is indicated by one trunk in figure 1 , but the vessels show much better in a mesial view. Mesial sections of pigs 5 to $6 \mathrm{~cm}$. long show that there is an abundant plexus of vessels arising from the stalk of the sac in the root of the neck near the place where the lymphatic sac connects with the vein. A few of these vessels follow the stalk or deep jugular trunk and are mentioned in connection with the lymphaties of the pharynx, more of them however follow the external jugular vein and connect with or help to form the superficial cervical plexus. In one specimen, one of these vessels connects directly with the external jugular vein instead of with the sac stalk. Thus the superficial cervical plexus can be said to arise not only from the apex of the sac but from the sac stalk as well or even by a variation directly from the veins. The plexus of vessels which follows the external jugular vein is very conspicuous in injections of eat embryos where the vein is entirely surrounded by a plexus of lymphaties.

In the pig $5.5 \mathrm{~cm}$. long the cranial end of the cervical plexus gives rise to three sets of lymphatics. First there are vessels growing behind the ear making a posterior auricular set. There is also a secondary plexus of vessels close to the main plexus which supplies (2) the temporal lymphatics and (3) the facial vessels. From the ventral border of the cervical plexus grow a group of deep submaxillary vessels and a very abundant plexus of vessels for the skin of the neck. The submaxillary plexus supplies the lower jaw and tongue and it has anastomoses both with the facial group and with the vessels from the anterior curvature of the sac. The submaxillary plexus may also connect with vessels along the internal jugular vein. The importance of these anastomoses between the primary groups of vessels cannot be emphasized too much.

The caudal end of the cervical plexus extends directly into the superficial thoracic vessels and supplies also the superficial lymphatics of the arm. These would show better in a ventral view than they do in the lateral one. In this point the drainage in the pig is different from that of the human embryo where the large thoracic vessels and the superficial vessels of the shoulder drain 
into the axillary vessels as can be seen in the figures from the Handbuch der Entwickelungsgeschichte quoted above. The pattern of the superficial vessels of the arm is to be seen in figure 5 of The American Journal of Anatomy, p. 188, volume 3, 1904.

The mesial view is better for the lymphatics of the anterior curvature of the sac. They show faintly in figure 4. Three groups of vessels can be injected from the anterior curvature in specimens 5 to $6 \mathrm{~cm}$. long. First an abundant group which extend to the wall of the pharynx, second vessels which extend along the sphenoid bone to the nasopharynx and third a small chain which grows outward toward the ear. It is probable that these are the lymphatics for the Eustachian tube. The anterior curvature is not the sole place of origin for the pharyngeal lymphaties for some injections show vessels which arise from the stalk of the sac low down in the neck, that is to say near the valveinto the vein. These vessels follow the course of the sac stalk, along the internal jugular vein to the wall of the pharynx. Some of the pharyngeal vessels anastomose with the superficial cervical plexus. Thus it may be said that there is an extensive budding of lymphaties from the veins of the neck. Almost all of these lymphatic buds make the deep jugular sac from which vessels arise in three places (1) from the apex, (2) from the anterior curvature and (3) from the stalk of the sac near the valve. These latter vessels are in the main deep lymphatics for the pharynx or superficial vessels which follow the external jugular vein. Occasionally a vessel arises independently from the external jugular vein itself. It may well be brought out here that none of the injections of the deep lymphaties has ever followed the arteries or veins in to the cranial cavity.

The origin of the lymphaties of the tongue is peculiar in that its vessels come from two sources. Lying beneath the mandible is the submaxillary plexus, and in the postcrior pharynx is the retropharyngeal plexus from the anterior curvature of the sac. The base of the tongue is situated between the two. Into it and into the adjoining part of the pharnyx grow ducts from each of these plexuses. At this stage of development the drainage is probably in both directions, but as the size of the cmbryo increases the more direct retropharyngeal route becomes the chief line, 
rather than the roundabout course through the submaxillary plexus. Thus it is clear that the deep lymphatics for the head and neck come from the sac stalk and its extension the anterior curvature. A part of them however, namely the submaxillary group come from the corvical plexus.

To sum up the relations of the superficial lymphatic vessels to the jugular lymph sac; three groups of vessels arise from the apex of the sac and therefore drain into the gland of the posterior triangle of the neck, the suprascapular vessels, the occipital vessels and the superficial cervical plexus. This latter extensive plexus has a double origin coming from the sac stalk as well or even by a variation directly from the vein. The stage of 5 to $6 \mathrm{~mm}$. represents the time when all of the primary superficial plexuses have been formed and have anastomosed with others in the skin so that there may be said to be one continuous plexus of lymphatic capillaries which covers the body. This plexus will become the deep subcutaneous plexus of lymphatic ducts. The development of valves in this plexus which now begins makes it impossible to obtain such extensive injections as can be made in embryos 5 to $6 \mathrm{~cm}$. long but the development of the valves tends again to bring out the primary plexuses which were lost by the development of the anastomoses.

From the anterior curvature of the sac and in part from the stalk of the sac develop the vessels for the pharynx and nose. These together with the submaxillary vessels from the cervical plexus represent the deep lymphatics for the head and neck.

All injections of later stages bring out the fact that the jugular lymph sac develops into two lymph glands and the deep jugular lymph trunk. In figure 4 is shown an injection of the deep lymphatics in the neck of a pig $7.5 \mathrm{~cm}$. long. It shows particularly well the position of the apex of the sac which is now a gland in the posterior triangle of the neck, between the sterno-cleido-mastoid and the trapezius muscles. The anterior curvature of the sac with some of the pharyngeal vessels lies under the sternocleido-mastord muscle. It is also a lymph gland. The sac stalk which is joined by the duct from the apex shows where it emerges from beneath the muscle. On the surface of the sterno-cleido- 
mastoid muscle and along its ventral border is the superficial cervical plexus. This is now one large gland. Its efferent vessels are the group of ducts to the apex of the sac and a large group to the sac stalk not injected in this specimen. They show well in other specimens connecting the cervical plexus with the stalk of the sac. They follow the external jugular vein and join the sac near the valve into the vein. The specimen shows some of the afferent vessels of the cervical plexus. Along two of the groups of afferent vessels namely the submaxillary vessels and along the facial vessels are developing lymph glands. This figure may well be compared with the injection of the lymph glands in the neck of a new born given as fig. 30 by Bartels in Das Lymphgefässsystem, in Bardeleben's Handbuch der Anatomie des Menschen. p. 103, 1909.

Injections of pigs $7 \mathrm{~cm}$. long show the same structural lines in the lymphatics as the pig measuring $5 \mathrm{~cm}$., with a few modifica tions. The relations of the jugular sacs are practically the same. One thing must, however, be noted, that the size of the sac has not changed much from that of the $5 \mathrm{~cm}$. pig. Consequently, the relative size of the sac being decreased, it does not occupy as extensive an area in the neck. The anterior curvature instead of being placed below the basi-sphenoid is below the atlas, and the apex lies in the plane between the third and fourth vertebrae instead of extending back to the fifth or sixth.

Another important morphological change is in the marked development of the lymph glands. The region of the sac between the anterior curvature and the apex having probably lost its function, more or less, as a line of drainage, has become considerably reduced in size, with three or four distinct constrictions. The beginning of this change was seen in the pig $5 \mathrm{~cm}$. long.

The third marked change in the sae is the shifting of the cross connection, which in earlier stages passes directly from the apex to the stalk. Instead of connecting by a short vessel with the stalk it passes parallel to it, a connection being finally established near the point where the stalk connects with the vein.

In embryos $8.5 \mathrm{~cm}$. long the sac stalk passes forward external to the internal jugular vein to terminate in a single large node 
posterior to the pharynx. From this node lymph vessels radiate to the deep structures of the head outside of the cranial cavity. A single large duct extends back to the apical node lying ventral to the trapezius muscle in the posterior triangle of the neck. This duct is plainly the atrophied remnant of the portion of the lymph sac lying between the apex and the anterior curvature. The cross connection between the apex and stalk lies along the transverse cervical vein and joins the sac stalk near the valve into the veins as in the embyro $7 \mathrm{~cm}$. long. The cervical plexus, consisting practically of large nodes, lies partly in front of the sternocleido-mastoid muscle and partly overlaps its anterior border. It is covered superficially by the panniculus carnosus muscle. There are two or three large nodes in front of the ear. Eight or ten ducts lead from the superficial cervical lymph nodes over the sterno-cleido-mastoid muscle, and pass to the primary apical node of the sac. Two or three vessels extend around the posterior margin of the masseter muscle, to the submaxillary lymph glands. In the injected specimens of this stage there is apparently only a single large duct from the cervical plexus along the external jugular vein to the root of the neck. Probably there are more which the injection did not reach. The retropharyngeal and part of the submaxillary vessels drain through the anterior curvature of the sac and the sac stalk. The superficial vessels of part of the head, the face, neck, thorax and arm drain through the superficial cervical glands either to the glands of the posterior triangle or directly to the sac stalk through the external jugularlymph trunks. The occipital and suprascapular vessels drain through the gland of the posterior triangle of the neck.

Dissections of the adult pig show that the gland which develops from the apex of the sac remains as a single gland in the posterior triangle of the neck. In one specimen it measured 2 by $3 \mathrm{~cm}$. long. The gland from the anterior curvature of the sac also remains a single gland. It is not as large as the apical gland measuring 1 by $2 \mathrm{~cm}$. It lies on the lateral surface of the internal jugular vein just dorsal to the pharynx. No other large gland is to be found along the internal jugular vein but there are a few small ones. It is clear that the gland of the posterior triangle 
of the neck and the pharyngeal gland of the pig are represented by groups of glands in the human being. The glands of the posterior triangle of the neck in a new born are well shown in the figure by Bartels, quoted above.

In the adult pig the superficial cervical plexus becomes a group of from twelve to eighteen glands lying along the external surface of the sterno-cleido-mastoid muscle. They are of all sizes varying from 1 by $2 \mathrm{~cm}$. long to one very large gland measuring 2 by 3 cm. just behind the ear near the origin of the sterno-cleido-mastoid muscle. This group of superficial glands clearly come from the superficial cervical plexus and the vessels which grow fromit. The efferent vessels from the superficial glands show well in Bartel's figure, that is those that run to the glands in the posterior triangle. The development of the superficial plexus leads one to expect that other efferent vessels follow the external jugular vein.

From this study it is possible to obtain a clear idea of all the lymph channels in the neck of the pig. From the apex of the jugular lymph sac develops a large node in the posterior triangle of the neck which receives the occipital, the suprascapular and the superficial cervical lymphatics, that is to say practically all of the superficial lymphatics for the anterior part of the body. The rest of the sac becomes the deep jugular lymph trunks and lymph glands of which the most cerebral one is by far the largest. The deep jugular glands drain the pharynx and Eustachian tube, the nose and in part the vessels of the tongue and lower jaw. The superficial cervical group of glands has the most complicated development. This group comes not only from the apex of the sac around the dorsal surface of the sterno-cleido-mastoid muscle but from the sac stalk or deep jugular trunk by vessels which follow the external jugular vein. It may also have a direct vessel from the veins. The superficial cervical plexus drains the posterior auricular region, the temporal, facial and cervical regions as well as the thoracic wall and the skin of the arm. The deep or axillary lymphatics of the arm have not yet been followed in the pig.

One of the most important points in this study is that there are primary plexuses of lymphatics for each region, that these primary 
plexuses are at first distinct connecting with each other only through the lymph sac which makes their common point of origin. Soon anastomoses between these primary plexuses connect them so abundantly that the entire surface of the body is covered with a primary and continuous plexus of lymphatics all of which can be injected from any one point. The subsequent development of valves follows the pattern of the original primary ducts as can be seen in figure 505 in the Handbuch der Entwicklungsgeschichte des Menschen, Keibel and Mall, p. 708, 1911, to such an extent that the primary lines of growth can be made out in injections. The study shows exactly what is meant by saying that the jugular lymph sac becomes transformed into lymph glands. The glands which arise from the sac itself we call primary lymph glands, those which develop on the vessels which grow from the sac may be called secondary and tertiary glands. The three kinds of glands are all shown in figure 4. Lymph glands develop at the points from which the peripheral lymphatics radiate out. 